PHYSICAL REVIEW D 96, 099906(E) (2017)

\title{
Erratum: Nucleon scalar and tensor charges using lattice QCD simulations at the physical value of the pion mass [Phys. Rev. D 95, 114514 (2017)]
}

\author{
C. Alexandrou, M. Constantinou, P. Dimopoulos, R. Frezzotti, K. Hadjiyiannakou, K. Jansen, C. Kallidonis, \\ B. Kostrzewa, G. Koutsou, M. Mangin-Brinet, A. Vaquero Avilès-Casco, and U. Wenger \\ (Received 27 October 2017; published 27 November 2017)
}

DOI: 10.1103/PhysRevD.96.099906

In this erratum, we give the corrected values of the disconnected contribution to the isoscalar, strange, and charm tensor charges, $g_{T}^{u+d}$ (disc.), $g_{T}^{s}$, and $g_{T}^{c}$ as explained in what follows. These corrected values are collected in Tables I and II, which replace the values presented in Tables VI and VII of the Paper.

TABLE I. Comparison of results for the disconnected contributions to the nucleon tensor charge from the plateau method, the summation method, and the two-state fit. The results extracted using the plateau method are presented for source-sink time separations $t_{s}=0.75 \mathrm{fm}$ to $t_{s}=1.31 \mathrm{fm}$. The final value selected for each observable is shown in Table II.

\begin{tabular}{|c|c|c|c|c|c|c|c|}
\hline \multirow{2}{*}{$\begin{array}{l}\text { Observable } \\
t_{s}(\mathrm{fm})\end{array}$} & \multicolumn{5}{|c|}{ Plateau value } & \multirow{2}{*}{$\begin{array}{c}\text { Two-state } \\
\text { fit } \\
\end{array}$} & \multirow{2}{*}{$\begin{array}{c}\text { Summation } \\
\text { method }\end{array}$} \\
\hline & 0.75 & 0.84 & 0.94 & 1.13 & 1.31 & & \\
\hline$g_{T}^{u+d}$ (disconnected) & $-0.0118(7)$ & $-0.0152(10)$ & $-0.0194(16)$ & $-0.0213(31)$ & $-0.0206(61)$ & $-0.0257(30)$ & $-0.0270(35)$ \\
\hline$g_{T}^{s}$ & $-0.00176(29)$ & $-0.00200(38)$ & $-0.00319(69)$ & $-0.00340(127)$ & $-0.00426(231)$ & $-0.00341(108)$ & $-0.00416(147)$ \\
\hline$g_{T}^{c}$ & $-0.00085(88)$ & $0.00010(137)$ & $-0.00263(269)$ & $-0.00731(439)$ & $-0.00750(666)$ & $-0.00226(398)$ & $-0.00062(502)$ \\
\hline
\end{tabular}

TABLE II. Results for the nucleon tensor charges, selected from the plateau fits. The error in the first set of parentheses is the statistical, in the second parenthesis is a systematic error due to the error in the determination of the renormalization function $Z_{T}^{\overline{\mathrm{MS}}}$, and in the third parenthesis is the systematic error taking into account excited state contaminations, which is taken as the difference in the mean values from the plateau and two-state fit methods.

\begin{tabular}{lcccc}
\hline \hline$g_{T}^{u-d}$ & $g_{T}^{u+d}($ connected $)$ & $g_{T}^{u+d}$ (disconnected) & $g_{T}^{s}$ & $g_{T}^{c}$ \\
\hline $1.004(21)(2)(0)$ & $0.582(16)(3)(1)$ & $-0.0213(31)(1)(44)$ & $-0.00319(69)(2)(22)$ & $-0.00263(269)(2)(37)$ \\
\hline \hline
\end{tabular}

Figures 1 and 2 replace Figs. 8 and 9, respectively, of the Paper. The corrected results for the disconnected contributions to the light and strange quarks are about 2 orders of magnitude smaller compared to the connected but show a clearly negative and nonzero signal. $g_{T}^{c}$ has also been corrected; however, this remains consistent with zero as in the Paper. Because of the small magnitude of the disconnected contributions, our conclusions regarding the sum of connected and disconnected contributions remain unchanged compared to the Paper.

Figure 3 compares $g_{T}^{u+d}$ (disc.) and $g_{T}^{s}$ to results from the PNDME group [1,2] at pion masses $m_{\pi} \sim 220 \mathrm{MeV}$ and $m_{\pi} \sim 310 \mathrm{MeV}$. For the disconnected contribution to $g_{T}^{u+d}$, our result is more negative compared to the value obtained at the smallest pion mass of PNDME and clearly nonzero. We note that the preliminary value of Ref. [3] agrees with our result. We note that the corrected value of $g_{T}^{s}$ is clearly nonzero and negative and remains consistent with the PNDME result that carries large errors.

Equation (1) replaces Eq. (33) of the Paper after using the correct values for the disconnected contributions; namely, we find

$g_{T}^{u}=0.782(16)(2)(13), \quad g_{T}^{d}=-0.219(10)(2)(13), \quad g_{T}^{s}=-0.0213(31)(1)(44), \quad g_{T}^{c}=-0.00263(269)(2)(37)$. 

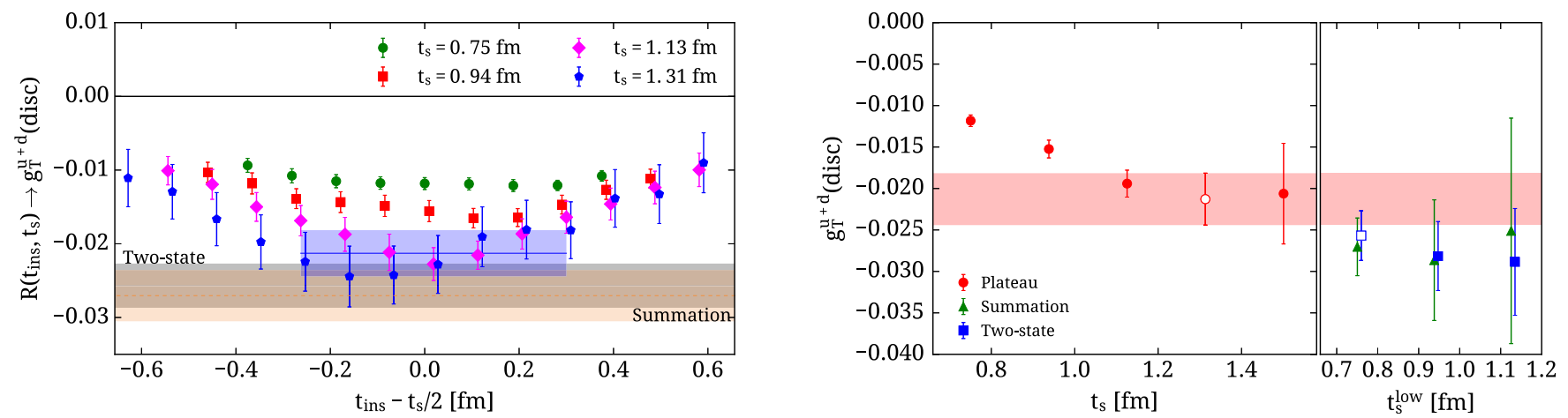

FIG. 1. Disconnected contributions to $g_{T}^{u+d}$. This replaces Fig. 8 of the Paper and follows the same notation.
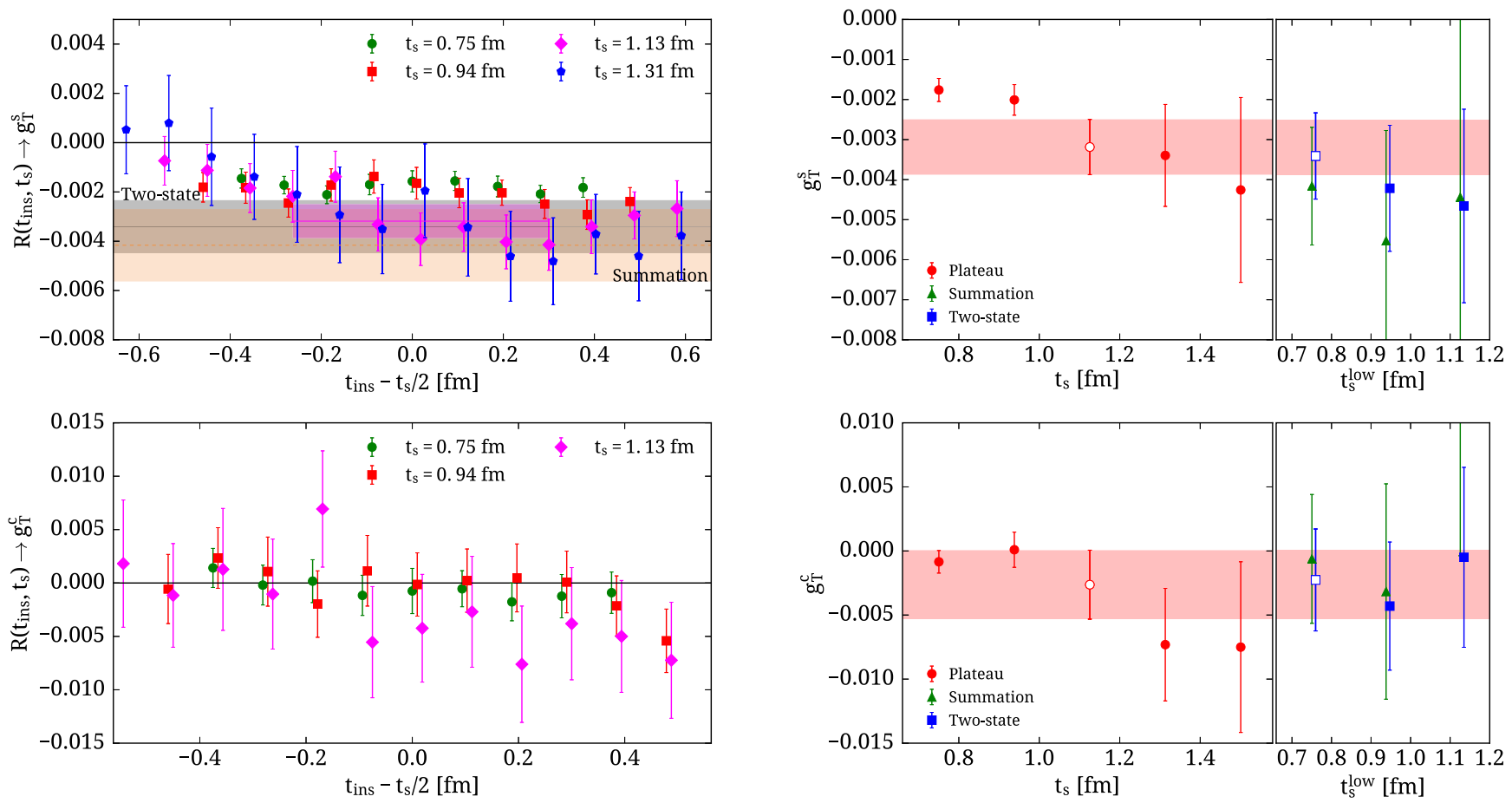

FIG. 2. The strange (top) and charm (bottom) tensor charges. The notation is as in Fig. 1. 

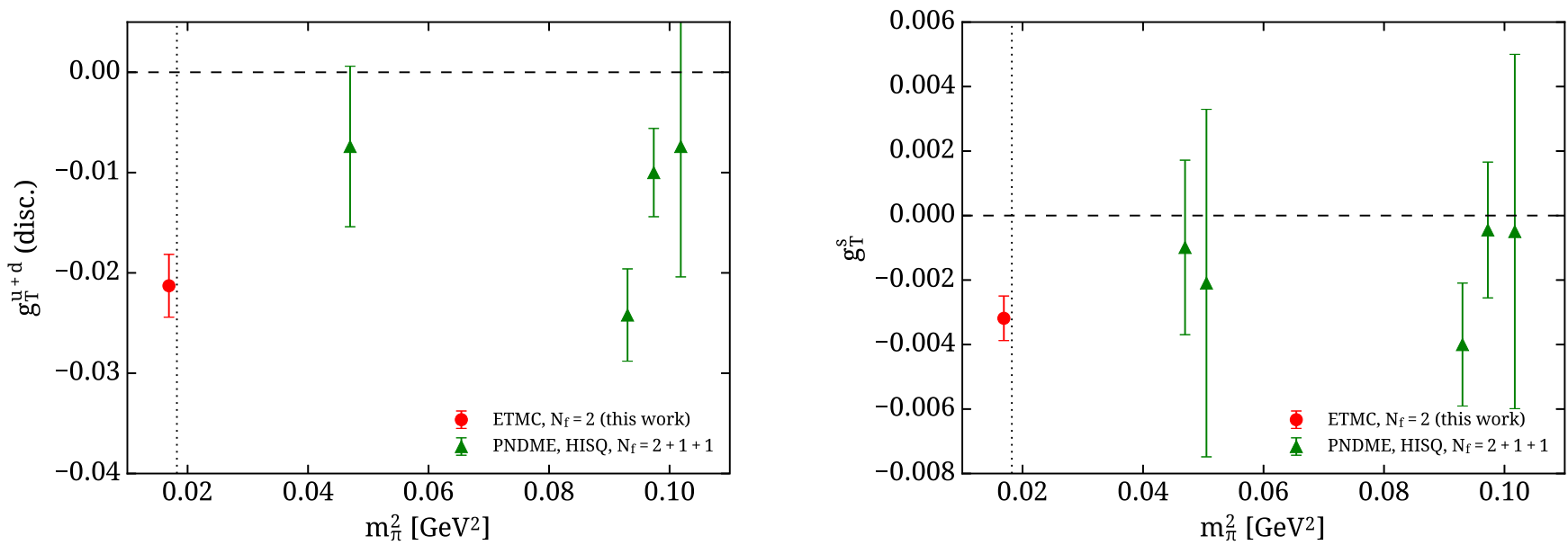

FIG. 3. Comparison of our results using the physical ensemble (red circles) for the disconnected contribution. Left: $g_{T}^{u+d}$ (replacing the right panel of Fig. 12 of the Paper). Right: $g_{T}^{s}$ (replacing the right panel Fig. 13 of the Paper). We include results from the PNDME Collaboration using clover valence fermions on an $N_{f}=2+1+1$ HISQ quark sea (green triangles) from Ref. [1].

In conclusion, our corrected results show that $g_{T}^{u+d}$ (disc.) and $g_{T}^{s}$ are nonzero and negative. They are, however, found to be small and do not change the overall conclusions of the Paper.

[1] T. Bhattacharya, V. Cirigliano, S. D. Cohen, R. Gupta, A. Joseph, H.-W. Lin, and B. Yoon, Iso-vector and iso-scalar tensor charges of the nucleon from lattice QCD, Phys. Rev. D 92, 094511 (2015).

[2] T. Bhattacharya, R. Gupta, and B. Yoon, Disconnected quark loop contributions to nucleon structure, Proc. Sci., LATTICE2014 (2014) 141.

[3] A. S. Gambhir et al., Algorithms for disconnected diagrams in lattice QCD, Proc. Sci., LATTICE2016 (2016) 265. 